\title{
THE FEASIBILITY OF INTERSPECIFIC RESCUE OF ENDOTHELIAL/HEMATOPOIETIC LINEAGE DEFICIENCY
}

Chongbei Zhao

\author{
Drs. John K. Critser, Kevin D. Wells and Elizabeth Bryda, \\ Dissertation Supervisors
}

\begin{abstract}
Here we propose to develop a mouse model which will establish "proof of principle" for future development of large animal (e.g. pig) models which could be used as actual human solid organ and blood donors as well as in pre-clinic trials. As a proof of concept, we intended to develop interspecific chimeras between mouse and rat to test the possibility that embryonic stem cells (ESCs) from one species are able to survive and develop in a blastocyst from a difference species. Then we intended to make a rat-mouse chimera with an endothelium/hematopoietic system from a rat genetic background and the other tissues and organs from a mouse genetic background. The first series of experiments of this thesis work were designed to test the feasibility for the interspecific chimeras (mouse-rat and rat-mouse) that can be made by ESC-blastocyst injection method. The second series of experiments of this thesis work were designed to test the feasibility of making such a chimera by using rat ESCs to rescue endothelial/hematopoietic system deficient mouse embryos that will otherwise die.
\end{abstract}

